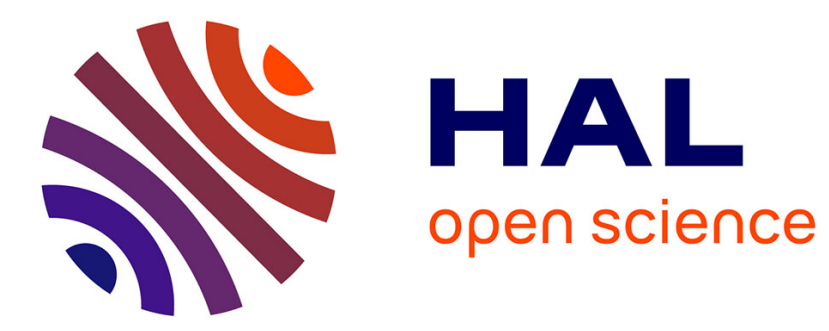

\title{
Quelques remarques sur "why" dans une perspective énonciative
}

Odile Blanvillain

\section{To cite this version:}

Odile Blanvillain. Quelques remarques sur "why" dans une perspective énonciative. Syntaxe et Sémantique, 2000, Connecteurs et Marqueurs de connexions, 1 (1), pp.183-212. 10.3917/ss.001.0183 . hal-01878837

\section{HAL Id: hal-01878837 \\ https://hal.science/hal-01878837}

Submitted on 7 Jan 2021

HAL is a multi-disciplinary open access archive for the deposit and dissemination of scientific research documents, whether they are published or not. The documents may come from teaching and research institutions in France or abroad, or from public or private research centers.
L'archive ouverte pluridisciplinaire HAL, est destinée au dépôt et à la diffusion de documents scientifiques de niveau recherche, publiés ou non, émanant des établissements d'enseignement et de recherche français ou étrangers, des laboratoires publics ou privés. 


\title{
Quelques remarques sur why dans une perspective énonciative
}

\author{
Odile BLANVILLAIN \\ Université de Caen - CRISCO
}

\begin{abstract}
Résumé: Cet article traite du marqueur why dans ses différents types d'environnements contextuels et tente d'en proposer une description unifiée, dans le cadre de la théorie des opérations énonciatives de Culioli. Il semble que l'on peut décrire why, de façon relativement féconde, comme la trace d'une indétermination qualitative de l'occurrence à laquelle il est associé. L'indétermination provient de son appartenance au paradigme $w h$-, et la spécificité de why au sein de ce paradigme correspond à son fonctionnement en tant que représentant du "fondement qualitatif» de l'occurrence (ce qui recouvre aussi bien cause que but, en tant qu'origine ou aboutissement notionnels). Ce fonctionnement introduit automatiquement un décalage entre la délimitation quantitative de l'occurrence et sa délimitation qualitative. Mais le décalage introduit n'est pas posé comme inhérent à l'occurrence, l'indétermination pouvant être levée et le rapport Qnt/Qlt stabilisé. Il introduit cependant la possibilité, souvent exploitée, de remettre en cause, au niveau qualitatif, l'occurrence considérée (d'où la variété des valeurs contextuelles de ce marqueur).
\end{abstract}

Summary: This article is a study of the marker why, and its goal is to propose a unified metalinguistic representation of this marker and its different contextual values (within the theoretical frame of the utterer-centered approach of Culioli). It appears that why can be described, in a rather productive way, as the trace of a qualitative indetermination of the occurrence to which it is associated. This indetermination (or underspecification) stems from the wh-paradigm itself, whereas the specificity of why can be assigned to its role as the image of the "qualitative foundation" of the occurrence (which can be equated with cause or goal, seen as notional origin or issue). This functioning leads automatically to a discrepancy (or gap) between the quantitative and qualitative determinations of the occurrence. But this gap is not inherent in the occurrence, since it can be filled. And yet, it allows to challenge the potentially well-founded nature (that is the qualitative foundation) of the occurrence concerned (thus the variety of contextual values mentioned before).

\section{Introduction}

Dans un précédent article, j'ai étudié les interrogatives en "Why should...? ", et en particulier l'interaction des marqueurs qui construisent l'interprétation plus ou moins polémique et rhétorique de ces énoncés. J'aimerais ici approfondir le rôle du marqueur why lui-même et étendre l'étude à ses différents types d'environnements contextuels. Mon 
but est de proposer une description unifiée du marqueur, en tant que trace d'une opération sous-jacente unique à laquelle on tentera d'associer une représentation métalinguistique, dans une perspective énonciative ${ }^{1}$.

\section{Why: représentant du fondement qualitatif de l'occurrence}

Je formulerai mon hypothèse de départ de la façon qui suit. Tout marqueur wh-constitue un domaine notionnel, et plus précisément l'image, le représentant d'un élément constitutif de l'énoncé: soit en tant que représentant d'un terme de la relation prédicative, soit en tant que marque d'un repérage énonciatif: il renvoie alors aux délimitations quantitative (Qnt) et qualitative (Qlt) de l'occurrence ${ }^{2}$ considérée. Who, par exemple, peut représenter le premier argument de la relation prédicative (C0), ou what le complément de rang un (C1). When et where, quant à eux, peuvent renvoyer au repérage spatio-temporel $(\mathrm{T})$ de la relation, et donc à son ancrage situationnel et à sa délimitation Qnt. De son côté, why est lié à l'autre aspect du repérage énonciatif ${ }^{3}, c^{\prime}$ està-dire à sa composante subjective (S), à la délimitation qualitative de la relation prédicative : il représente le «fondement qualitatif » de l'occurrence considérée ${ }^{4}$.

1. Cette étude s'inscrit plus précisément dans le cadre de la théorie des opérations énonciatives de Culioli.

2. Pour une définition d'occurrence et de délimitations quantitative et qualitative de l'occurrence, cf. Franckel \& Lebaud 1990 (annexe) : «Une notion ne peut être appréhendée qu'à travers des occurrences de cette notion, ces occurrences constituant à la fois le point d'ancrage de la notion (ce qui la manifeste et permet d'en parler) et son incarnation qualitative [...]. Une occurrence renvoie à un événement énonciatif qui opère sur la notion deux types de délimitation qui s'articulent de façon variable. " Ces deux délimitations y sont définies de la façon suivante : la première correspond à l'ancrage situationnel de la notion (Qnt) et la seconde à sa délimitation qualitative (Qlt). Cette dernière permet « de distinguer ce qui est X de ce qui est qualitativement autre que $X$. Elle est associée à la structuration notionnelle d'une notion ».

3. Aux deux modes de délimitation d'une notion correspondent deux modes de repérage. Ibid.: «Pour repérer une notion, il faut : - d'une part la situer, lui assigner des coordonnées dans le temps et dans l'espace, l'ancrer à un repère $(T)$. Il s'agit de déterminations d'ordre Qnt; - d'autre part la spécifier, c'est-à-dire la rapporter à un type. [...] Il s'agit de déterminations d'ordre Qlt. Cet étalonnage qualitatif, associable à une valuation (plus ou moins conforme, adéquat, favorable) est une opération nécessairement rapportée à un sujet : la structuration notionnelle ne s'opère qu'en référence à un repère subjectif $(S)$, instance subjective. "

4. Notons immédiatement qu'il ne s'agit pas d'une classification où l'on étiquetterait chaque marqueur de façon définitive, mais d'une proposition de représentation d'un comportement, c'est-à-dire d'un fonctionnement en contexte. Il semble par exemple que what puisse fonctionner comme l'image d'un terme de la relation, mais également comme le représentant de la délimitation quantitative de l'occurrence. 
Le Petit Robert définit «fondement " par « ce qui détermine l'assentiment légitimé de l'esprit; fait justificatif d'une croyance, d'une réalité. [...] V. motif, raison, sujet», également comme "point de départ logique [...]. V. principe». J'ai associé à l'idée de fondement le terme de «qualitatif» dans la mesure où le fondement de l'occurrence participe à la structuration qualitative, notionnelle de l'occurrence pour en faire une «bonne» occurrence, l'occurrence «qu'il fallait» (valuée positivement par une instance subjective ou objectivement en adéquation à une situation), ou encore une "vraie » occurrence (centrée, rapportée à un type). Le fondement qualitatif d'une occurrence constitue une assise notionnelle possible de l'occurrence, ce qui lui donne du «sens ». Ceci apparaît assez clairement dans l'énoncé suivant :

(1) The fundamental question which the form-critic asks is "Why was the story told?" or "What is the point of the story?"

H. Gardner, Business of Criticism, ii. ii, p. $105\left[O E D^{5}\right]$

où What is the point of the story?, qui vient expliciter la question en why, montre bien que l'on s'interroge ici sur ce qui donne un sens à l'histoire. Dans les exemples (2) et (3) :

(2) It is, as I say, the why of crime that interests nowadays.

A. Christie, Murder in Retrospect, p. 49 [Deléchelle 1989]

(3) The why of the symptom, its tendency, is always an endo-psychic process.

Freud, Introd. Lect. Psycho-anal., trad. J. Riviere (1922), p. 240 [OED]

the why of crime s'interprète comme les «motivations subjectives» du crime (ce qui fonde l'occurrence pour le sujet qui en est à la source). Et the why of the symptom correspond à ce qui en est à l'origine et l'explique.

On retrouve bien sûr avec l'idée d'origine ou de motivation subjective, l'idée de déclenchement "causal ». L'interprétation donnée à why peut varier selon le contexte (relations intersubjectives, types de verbe, relations primitives des termes en présence, etc.) comme on pourra le constater au fur et à mesure de cette étude. Les termes les plus fréquemment employés à son propos sont ceux de "cause», "reason", "motive», "intention» et "purpose»". Mais on pourra noter que, quelle que soit

5. Oxford English Dictionary, 1992 (cité désormais OED).

6. Cf., par exemple, Quirk et al. 1995, p. 695: Phrases of cause, reason, and motive answer the question "Why...?", et l'OED (article why I. 1. a) : For what reason? from what cause or motive? for what purpose? wherefore? En ce qui concerne particulièrement le but, G. Deléchelle (1989, p. 199) note que "Aristote incluait déjà la cause finale dans ses quatre types de cause", et É. Gilbert (1999, p. 115) que cause (ou «origine notionnelle») et but (ou "aboutissement notionnel») ne se distinguent fondamentalement que par une différence d'orientation. 
l'interprétation choisie, why pourra toujours se définir comme le représentant du «fondement qualitatif» de l'occurrence considérée.

On peut relier l'idée de "représentation" (les éléments wh-sont tous des représentants) à l'idée d' «indétermination » (ou de détermination minimale) comme le fait $\mathrm{H}$. Adamczewski ${ }^{7}$, les éléments $w$ h-correspondant selon lui à des «formes vides en attente de remplissage, ce qui explique d'ailleurs à la fois leur emploi comme pronoms interrogatifs... et comme pronoms relatifs (où le remplissage est le fait de l'antécédent!)». L'idée de "parcours», proposée par Culioli, peut être ici également utilisée. En effet, l'élément wh-en tant que représentant du domaine des valeurs possibles et imaginables assignables au terme représenté correspond à une classe d'occurrences, qui peut à ce titre être parcourue. Le parcours de la classe pourra se voir attribué une issue différente selon le contexte.

On peut faire ressortir dès maintenant, à partir de ces idées fondamentales dans le fonctionnement des marqueurs wh- (représentation et parcours, ou indétermination), la question du «décalage» associé à why. On a dit que why était le représentant de ce qui fonde qualitativement l'occurrence, mais la nécessité même de représenter suppose une indétermination à ce niveau. L'occurrence se voit ainsi privée de son $\operatorname{site}^{8}$ (au niveau qualitatif, notionnel), ce qui introduit automatiquement un décalage entre la délimitation quantitative de l'occurrence et sa délimitation qualitative.

7. Cf. Adamczewski 1982, p. 324. Le même auteur note (p. 328-329) que « wh- [...] s'oppose à th-comme marque d'indétermination ", that correspondant - selon la terminologie de l'auteur - à du «thématique " (phase 2), et who/ which à du «rhématique " (phase 1). Cette différence de statut des deux morphèmes quant à l'« indétermination " me semble pouvoir être directement liée à la différence entre "fléchage» et "parcours", en termes culioliens.

8. À propos de "site", on pourra remarquer le lien qui semble exister entre l'emploi d'un élément $w$ h- et la construction d'un «repère constitutif». Selon Culioli (1982): "construire le repère constitutif, c'est construire le domaine organisateur de l'énoncé ", ou encore (Culioli 1990a, p. 120) : «la construction d'un repère constitutif se ramène à la construction d'un terme distingué qui va servir de site à une relation prédicative. » Or, il semble que l'emploi d'un élément $w$ h-entrâne assez systématiquement une organisation de l'occurrence considérée autour du domaine notionnel correspondant à cet élément $w h$ - (comme l'indique d'ailleurs sa position initiale). L'élément $w h$-correspond toujours au terme distingué par son indétermination même et le fait que son domaine notionnel fait l'objet d'un parcours. Ceci s'oppose à l'idée qu'un "repère" (constitutif ou autre) doit être stable, mais on pourrait considérer que l'élément $w$ h-constitue une sorte de site potentiel autour duquel s'organise l'occurrence en attente de stabilisation. 
É. Gilbert ${ }^{9}$ parle, à propos de for, d'un hiatus entre les délimitations quantitative et qualitative de l'occurrence considérée. Je propose le terme de décalage pour why dans la mesure où l'indétermination qualitative qu' il marque permet d'introduire un hiatus, mais ce dernier peut être comblé (il suffit pour cela que soit levée l'indétermination). Why (contrairement à for) n'est donc pas incompatible avec l'idée d'adéquation des délimitations Qnt et Qlt de l'occurrence. S'il est lié à l'idée de décalage par le fait de sa fonction de représentation et du type de domaine qu'il représente, why ne pose cependant pas le hiatus comme inhérent à l'occurrence, puisqu'il y a toujours possibilité de faire disparaitre le décalage en instanciant la variable $w$ h- (en lui assignant une valeur donnée, ce qui revient à fournir une issue au parcours). Ceci aura pour effet de (re)donner à l'occurrence un statut d'occurrence notionnelle, ou comme on l'a dit précédemment, d'en faire une «bonne » occurrence. On retrouvera ce jeu entre stabilisation et déstabilisation du rapport Qnt/Qlt de l'occurrence dans tous les emplois de why, comme je vais essayer de le montrer maintenant.

\section{Why relatif}

Dans son emploi relatif, why représente le fondement qualitatif de la relation prédicative correspondant à la subordonnée relative. Le fondement de l'occurrence considérée reste indéterminé au sein de la relative, mais on pourrait qualifier ce décalage de «local» dans la mesure où il s'efface «ailleurs" (puisque l'instanciation se fait dans l'énoncé ou le contexte ${ }^{10}$ qui fournit donc l'issue du parcours). On peut illustrer ce fonctionnement par les exemples suivants:

(4) Roy Taylor, deputy chairman of the Magistrates Association, says there are two reasons why shoplifters have become more active. "There is more pressure, through shop displays and advertising, for people to have things, so they steal them," he says. "And then there are young people who want a challenge. They don't need what they steal and they don't profit from it. It's just a dare."

R. Daft, "Meet the middle-class shoplifter", Times, 21 février 1998, p. 19

9. Cf. Gilbert 1999. Ce hiatus ne concerne pas les délimitations quantitative et qualitative de l'occurrence (c'est-à-dire sa structuration interne) dans tous les emplois de for (cf. l'article d'Éric Gilbert, dans ce recueil). Mais c'est le cas (entre autres) pour sa valeur causale.

10. Je dis que c'est l'énoncé ou le contexte qui lève l'indétermination et non simplement l'antécédent car remplacer why par reason ne comble pas véritablement le déficit informationnel. (NB : on peut, à la suite de G. Deléchelle (1989, p. 222), noter le caractère privilégié du terme reason qui est le seul antécédent possible de why relatif, à l'exclusion de *cause, *motive ou *explanation). 
(5) He could think of one vital reason why this killer should want to avoid any suggestion that Digby had killed himself through remorse or had been in any way concerned in his brother's death.

P.D. James, Unnatural Causes, 1967, p. 170

Why peut inclure son antécédent (cas des relatives nominales «à antécédent amalgamé»), comme en (6) :

(6) The project, chronicled in Richard Preston's book American Steel, was so controversial that Bethlehem Steel commissioned an inch-thick report on why it wouldn't work. But it did work.

D. Kadec, "Lunch-Pail», Time Magazine, 23 mars 1998, p. 11

Dans les exemples suivants, il y a identification (ou tentative d'identification) du fondement notionnel de l'occurrence considérée (ce qui signifie que le parcours trouve une issue et que l'occurrence est stabilisée) :

(7) To hear Frederic Whitehurst tell it, his goal is to serve his country. That is why he volunteered for combat in Vietnam, where he earned four bronze stars, and that is why in 1982, armed with a Ph. D. in chemistry from Duke, he joined the F.B.I.

E. Gleick, "Explosive Charges », Time Magazine, 4 novembre 1996

(8) Big daddy: Son, you know you got a real liquor problem?

Brick: Yes, sir, yes, I know.

Big daddy: Is that why you quit sports-announcing, because of this liquor problem?

Brick: Yes, sir, yes, sir, I guess so.

T. Williams, Cat on a Hot Tin Roof, 1957, p. 58

Mais on trouve également des exemples où le décalage est maintenu, tel qu'en (9) :

(9) "The Bradys don't have that much money to scatter at the Royal Hotel."

"They don't mind that. It's just one day."

"There'll be no hotels. We are too old and poor for that."

"It'll be thought strange. Everybody does it."

"Because everybody goes and jumps in the river is no reason why we have to go and jump in the same river."

J. Mc Mahern, Amongst Women, 1990, p. 38

Dans cet exemple, une raison a été retenue (Because everybody goes and jumps in the river) pour être aussitôt réfutée ([it $]$ is no reason). On trouve d'ailleurs de nombreux exemples dont le but est de poser l'absence de raison:

(10) Such was the strength of the instinct that they felt that they could force their beloved to remain in life if only they could, together, turn his will around. Since they had the power of birth there was no reason why they couldn't will this life free of death. For the first time in his life Moran began to fear them.

J. Mc Mahern, Amongst Women, 1990, p. 178 
(11) "I think of them as old women," he says, "though there's no reason why they shouldn't have been men, I suppose. They could be anything as long as they were willing to eat the sins [...]"

M. Atwood, "The Sin Eater», in Dancing Girls and Other Stories, 1977, p. 214

On pourra mettre ces énoncés en parallèle avec les suivants ${ }^{11}$ :

(12) I can't see Digby acting the butcher and I can't see why he should.

P.D. James, Unnatural Causes, 1967, p. 77

(13) And Michael Bonallack, secretary of the Royal and Ancient, golfs rule-making body, says: "Every other major sport is in the Olympics, I don't see why we should be left out."

"Augusta is ready to open its gates", The European, 29 octobre 1992, p. 32

Ces derniers peuvent eux-mêmes être comparés aux interrogatives directes en "Why should...? ", généralement qualifiées de rhétoriques et où ressort facilement l'idée de décalage, de remise en cause du bienfondé de l'occurrence.

\section{Why interrogatif}

Le marqueur why est souvent associé à la forme interrogative ${ }^{12}$ et garde même ce sens dans certains de ses emplois comme substantif:

(14) She could supply the ready "because" to many of the old philosopher's "whys" [OED, article "Why"]

11. Ces énoncés correspondent à des interrogatives indirectes, de même que les suivants : - [...] I used to wonder why I had worked so hard to reach a place where a wide range of people from taxi drivers, hotel managers, and government officials to muggers were bent on ripping off or robbing westerners. (A. Krushelnycky, «Moscow», The European, 1997)

- I don't know what I did to deserve it. I don't know why things can't be the same in this house as in every other house in the country. I don't know why it is always me that has to be singled out. (J. Mc Gahern, Amongst Women, 1990)

- Look here, Komarov [...] I cannot understand who else here might want this book; certainly none of my students; and if it is you, I cannot understand why you should want it anyway. (V. Nabokov, Pnin, 1960)

Mais comme le signalent R. Quirk et al. (1995, \$15.8-9, p. 1056-1061), il est parfois difficile de distinguer entre relative nominale et interrogative indirecte.

12. Lorsque D. Bolinger compare that et which relatifs, il remarque que that est fondamentalement un démonstratif, alors que which (et j'ajoute: tout relatif $w h$-) est fondamentalement un interrogatif (cf. l'article très éclairant de S. Gresset (1984) concernant les relatifs that et which qui rappelle, entre autres, les hypothèses de D. Bolinger à ce sujet). 
(15) For every Why we must have a Wherefore.

[OED, article "Why»]

\subsection{De l'indétermination qualitative à la valuation négative}

La forme interrogative représente sans doute la forme la plus évidente de "parcours». Dans le cas correspondant aux demandes d'information (ou "vraies" questions), l'indétermination qualitative entraîne un parcours sans issue, d'où le recours à autrui, qui permettra d'instancier le domaine des possibles ${ }^{13}$. L'absence de stabilisation de l'occurrence peut n'être due qu'à l'ignorance dans laquelle se trouve le locuteur. On peut considérer que le décalage n'est plus «local ", mais "temporaire" (dans l'attente de la réponse qui le fera disparaître en fournissant un site à l'occurrence).

Certains verbes supposent automatiquement une cause, dans la mesure où, selon les termes d'É. Gilbert ${ }^{14}$, ils représentent eux-mêmes «l'issue, le résultat situationnel d'une origine notionnelle préexistante». La question en why visera donc à établir cette cause ou "origine notionnelle», (pré)supposée existante mais inconnue du locuteur. La réponse attendue permettra d'annuler le décalage "artificiel » dû à l'ignorance du locuteur, pour rendre à l'occurrence son statut d'occurrence notionnelle :

(16) "Why did he arrest you?" "Oh, I dropped a cigarette out here in summer an' started a fire, an' he held me under the fire law."

H. Titus, Timber iii, 1922, p. 32 [OED]

Ce qui fait de l'occurrence considérée une «vraie» occurrence de la notion, c'est ce qui justifie son existence : l'arrestation était "fondée " (il n'a pas été arrêté "pour rien»). On peut donc avoir une véritable demande d'information avec ce type de verbes.

La recherche du fondement notionnel de l'occurrence peut également correspondre à la recherche des motivations du sujet. Ce qui est en question, c'est alors ce qui fait de l'occurrence une «bonne» occurrence pour le sujet de l'énoncé, source de l'action (sa motivation ou son but, par exemple) :

(17) Q. Why did you go into the apartment?

A. To rip it off.

13. Cf. Culioli 1990b, p. 164.

14. Cf. Gilbert 1999, p. 112, qui regroupe un ensemble de verbes : "punish, condemn, fine, indict, reward, recompense, ridicule, apologize, thank, criticize, beat, kill, berate, convict, arrest, bless, etc.", verbes qui sont "tous compatibles avec l'existence d'une délimitation qualitative préconstruite, susceptible de fonder la validation de la relation prédicative». 
Q. To burglarize it?

A. Yes.

E. McBain, Sadie when she Died iii, 1972, p. 30 [OED]

Cependant, on sait que la forme interrogative peut correspondre à une forme de rejet (interrogative «rhétorique»). Dans ce cas, il ne s'agit pas d'attendre d'autrui qu'il apporte une issue au parcours (et confirme le "bien-fondé" de l'occurrence), mais bien au contraire de marquer qu'il n'existe aucun fondement qualitatif possible de l'occurrence, ce qui se traduira naturellement par une valuation négative. Mais quelle que soit l'orientation de la question (vraie question ou question rhétorique), l'interrogative en why met toujours en jeu la structuration notionnelle de l'occurrence considérée : qu'il s'agisse d'en faire une "bonne " occurrence (en levant l'indétermination qualitative), ou de l'associer à une valuation subjective négative (lui déniant ainsi le statut d'occurrence notionnelle). Ceci explique sans doute la possibilité du continuum observé dans le statut des interrogatives en why, entre les «vraies» questions (qui attendent une réponse et sont relativement neutres au niveau des relations intersubjectives) et les interrogatives "rhétoriques » (qui n'appellent aucune réponse et font apparaître clairement la position du locuteur/énonciateur ${ }^{15}$ ), entre la recherche objective du fondement notionnel de l'occurrence et sa remise en cause. En effet, le locuteur peut également attendre une réponse tout en faisant transparaitre son point de vue négatif sur la question (il peut laisser ouverte la possibilité de lever l'indétermination qualitative de l'occurrence tout en la valuant négativement). Voici simplement quelques exemples de ces interrogatives «argumentatives» (simplement polémiques et/ou rhétoriques), où le locuteur - même lorsqu'il attend une réponse - fait transparaître clairement son point de vue (l'énoncé pouvant à chaque fois être glosé par : "you shouldn't do/have done that») :

(18) Big Daddy: Why do you drink?

Brick: Don't know, give me my crutch!

Big Daddy: You better think why you drink or give up drinking!

Brick: Will you please give me my crutch so I can get up off this floor?

Big Daddy: First you answer my question. Why do you drink? Why are you throwing your life away, boy, like somethin' disgusting you picked up on the street?

T. Williams, Cat on a Hot Tin Roof, 1957, p. 69-70

15. On peut se demander ici s'il faut parler de locuteur ou d'énonciateur: locuteur semble plus approprié du fait de la tournure interrogative (liée à l'absence de prise en charge), mais énonciateur semble également envisageable quand l'interrogative est rhétorique (du fait d'une certaine prise en charge de la valuation négative). 
(19) Margaret: [...] Why are you looking at me like that?

Brick [whistling softly, now]: Like what, Maggie?

Margaret [intensely, fearfully]: The way y' were lookin' at me just now, befo' I caught your eye in the mirror and you started $t^{\prime}$ whistle!

T. Williams, Cat on a Hot Tin Roof, 1957, p. 23-24

(20) For crying out loud, why did you do it?

R. West, Black Lamb ii, 1941, p. 156 [OED]

Notons également que si la question peut être polémique, il en va de même de la réponse. Lorsqu'une réponse n'en est pas vraiment une, le hiatus ne se trouve que superficiellement comblé, comme le montrent les exemples suivants:

(21) Margaret: Well, I just remarked that one of th'no-neck monsters messed up $m$ 'lovely lace dress so I got t'cha-a-ange...

[she opens and kicks shut drawers of the dresser]

Brick: Why d'you call Gooper's kiddies no-neck monsters?

Margaret: Because they've got no necks! Isn't that a good enough reason?

T. Williams, Cat on a Hot Tin Roof, 1957, p. 17

(22) Mae: Maggie?

[Margaret turns with a smile]

Why are you so catty?

Margaret: Because I'm a cat!

T. Williams, Cat on a Hot Tin Roof, 1957, p. 30

On peut ainsi obtenir toute une variété de jeux sur l'orientation de la réponse, avec éventuellement effet de "rebuffade»" ${ }^{16}$ On peut, par exemple, ajouter les cas où la réponse se limite à "because!». Ce type de réponse, immédiatement ressentie comme polémique, marque que l'occurrence est bien fondée notionnellement, mais sans précision de ce qui la fonde, la justifie :

(23) "Why didn't you leave the bottle?" "Because!" I said shortly. I wasn't going to explain my feelings on the matter.

M. Carroll, Dead Trouble x, 1968, p. 175 [OED]

(24) "Why do you go out with him, Helen?" "Because." Helen didn't mean to be cheeky.

C. Fremlin, Prisoner's Base vii, 1967, p. 48 [OED]

On pourra remarquer que ces jeux sur l'ambiguïté éventuelle de la question ou sur la réponse sont dans tous les cas permis par l'introduction initiale d'un décalage au niveau des délimitations quantitative et qualitative de l'occurrence.

16. Au sens de Milner 1983. 


\subsection{Le cas de "Why + mention de la notion?"}

Si la positivité ${ }^{17}$ de l'occurrence est de type "positivité première", c'est-à-dire si why porte sur la simple mention d'une notion (lexème verbal, nominal, adjectival, etc.), l'interrogative pourra s'interpréter comme la recherche de ce qui fait de cette occurrence le "bon choix". Ceci dit le passage ${ }^{18}$ d'une positivité Qnt ou Qlt au type de positivité conférée par la mention met déjà en évidence le décalage instauré par le locuteur :

(25) Oh well. What can she expect? Why come, anyway? Should have stayed at home. So he doesn't want to sleep in the open, on the deck. Under the stars. All right. No stars, then.

N. Gordimer, «Spoils», The Minerva Book of Short Stories 1, 1988, p. 84

(26) "Why high hat me?" he complains. "I'm harmless and I may be able to do you a lot of good."

H.C. Witwer, Cosmopolitan, 68/1, Avril 1924 [OED]

(27) "Why pay top money for information and a photograph of a child whose surname they don't know?" "Perhaps they want a penpal?"

P. B. Yuill, Hazell plays Solomon ii, 1974, p. 25 [OED]

17. Les deux modes de délimitation Qnt et Qlt correspondent à deux formes de positivité d'une occurrence (cf. Franckel \& Lebaud 1990, annexe) : la "positivité conférée par l'ancrage situationnel. Il s'agit d'une positivité d'ordre Qnt» et la positivité qualitative, qui «renvoie: soit à l'idée de bonne valeur. Il s'agit d'une positivité liée à une valuation et par conséquent à un repérage subjectif. [...] soit à l'idée de centrage ou de conformité à un type ». Les auteurs définissent également un troisième type de positivité: celle "conférée à une notion par sa simple mention. C'est par exemple le cas de les fantômes dans un exemple comme Les fantômes, ça n'existe pas". Ils rappellent également que "la mention d'un domaine correspond au point de départ d'une bifurcation dont l'une des branches correspond à l'existence (p), l'autre à l'inexistence $\left(\mathrm{p}^{\prime}\right)$. Cette position décrochée peut également correspondre au point de départ d'une bifurcation conduisant à I d'une part, à E d'autre part». Cette position décrochée sera notée "hors $p$ par rapport à la délimitation situationnelle » et IE « relativement à la délimitation qualitative, c'est-à-dire à la structuration du domaine notionnel en extérieur/intérieur». I correspond à l'intérieur du domaine notionnel et $\mathrm{E}$ à son extérieur. J'utiliserai donc de préférence $\mathrm{p} / \mathrm{p}$ ' quand l'accent sera mis sur la validation de la relation, sa délimitation quantitative (son repérage situationnel) et I/E quand l'accent sera mis sur la valuation de la relation, sa délimitation qualitative (son repérage subjectif).

18. Il ne faut pas oublier que la mention correspond à un préconstruit (toute interrogative ouverte supposant un préconstruit, même si la préconstruction est fictive, cf. Blanvillain 1997) et qu'elle renvoie donc à une occurrence "initiale» dont la positivité peut être de type quantitative ou qualitative. 
D'ailleurs ce type d'énoncés s'interprète souvent comme une remise en cause du choix (préconstruit ${ }^{19}$ ) de la mention : soit qu'il est «mauvais» (valué négativement), décalé par rapport à l'attente du locuteur, soit qu'il est inadéquat, décalé par rapport à la situation. On part de p (ou de I, selon le type de positivité, c'est-à-dire selon que l'on s'intéresse particulièrement à l'aspect validation de la relation, ou à sa valuation). L'interrogative en why ramène cette occurrence de départ à sa simple mention. Cela peut s'exprimer comme le retour en un lieu d'où le choix est encore possible, et métalinguistiquement comme le repositionnement au point de départ de la bifurcation (en hors $p$ ou en IE) qui mène soit à $\mathrm{p}(\mathrm{I})$, soit à p' (E). À partir de ce point de départ, on peut à nouveau envisager la validation ou la valuation de l'occurrence.

Les interrogatives du type "Why not + base verbale? ? fonctionnent globalement comme celles du type: "Why + base verbale? ", si ce n'est que la forme négative pousse encore davantage vers l'interprétation rhétorique $^{20}$. De plus, la présence de not semble faire pencher pour l'interprétation quantitative (pourquoi ne pas valider p? , plutôt que pourquoi ne pas choisir I?), d'où l'interprétation en termes de proposition de faire. Le parcours sur ce qui pourrait déclencher le choix de not $p$ aboutissant à l'ensemble vide (il n'y a pas de raison pour ne pas), on aboutit ainsi à la suggestion de valider $\mathrm{p}$ :

(28) "Why not let's offer them to one of the big stores in town as a Christmas show?" she suggested.

G. Durrell, A Zoo in my Luggage, 1960, p. 171

(29) He said: "So long, sister. We'll see you in a month and three days." And she said: "Off you go, boys - into the wild blue yonder." There was an awkward silence after that, and since it made me feel uncomfortable, I opened my big mouth and said: "What do you say, ma'am? Why not hop in the car and come with us?"

P. Auster, Mr. Vertigo, 1994, p. 119

19. Notons que dans ce type d'énoncés, toute trace de la préconstruction est effacée. On perd tout le repérage énonciatif: non seulement on passe d'une positivité Qnt ou Qlt de l'occurrence à une positivité dite "première", mais on perd la relation prédicative initiale pour n'en garder qu'un terme (le verbe dans le cas de "Why + base verbale?»). De ce fait, un énoncé du type "Why + base verbale?", abordé hors contexte, sera naturellement interprété comme la mise en cause de la mention d'une notion simplement envisagée (et non pas validée, même si c'est le cas).

20. Il semble que si le locuteur attend une réponse, il utilisera plutôt la forme finie : "Why don't you...?" 
Dans les énoncés suivants, le choix mentionné (et critiqué) est mis explicitement en parallèle avec un autre possible (valué positivement) ${ }^{21}$ :

(30) Why buy soap in packages when you can buy it cheaper "unwrapped"?

Daily Colonist (Victoria), 8/1, 20 mars 1921 [OED]

(31) Why pay the five per cent front end charge, plus an annual management charge for something you can do yourself.

Times, 14, 9 mars 1985 [OED]

Le recours à la base verbale est ici particulièrement efficace (il s'agit de slogans publicitaires) puisqu'elle permet d'envisager à l'avance un choix, et de le rejeter en faveur d'un autre. Avec la négation, on aura une "proposition de faire", mise en avant explicitement par rapport à un autre choix possible, mais valué négativement :

(32) Why not take your old non-returnable glass bottles to your local bottle bank instead of throwing them away?

Which?, 355/3, août 1984 [OED]

(33) Why not approach the institutional or wholesale money market for funds in large tranches rather than continue with the costly exercise of collecting money from a multitude of smaller savers?

Times, 19/4, 18 janvier 1980 [OED]

La mention considérée peut également être exprimée par un syntagme (nominal ou autre) ${ }^{22}$. Le terme utilisé peut traduire une attitude ou l'état de la situation observée (d'où les marques éventuelles de fléchage situationnel) :

(34) Why so much chestiness? I haven't sold anything to any of you, have I?

O. Johnson, Varmint xiii, 1910, p. 181 [OED]

21. Ce décalage explicite (marqué par when, instead, rather than, un comparatif, etc.) est fréquent dans les interrogatives en "Why + mention?", mais on le retrouve avec tous les types d'interrogatives en $w h y$. En voici quelques exemples:

- I asked a prominent East German lawyer why, rather than sneer about "revanchists", they did not support this attempt to convict Nazi criminals. (New Statesman, 1965, in $O E D$ )

- Why, oh, why, do little boys (and big ones) rush to a jobnny when nature provides opportunity everywhere? (D. Conover, One Man's Island, 1971, in OED)

- Said a jobless Hamilton steelworker, father of six children: "Why should I sweat for $\$ 40$ a week? I'm getting more than that from the pogey, the welfare and the baby bonus." (Times, 1961, in OED)

22. Le fonctionnement étant identique comme le montre bien l'exemple suivant : - "[...]. She was pretty determined - wasn't she - to work in those particular Chambers. She was doing perfectly well with Sir Roderick Matthews. Why change? Why Pawlet Court?" (P.D. James, A Certain Justice, 1997) 
(35) "Why the rush?" "Well, I don't know, darling. She just said, could we come as soon as poss."

D. Francis, Smokescreen ii, 1972, p. 30 [OED]

(36) If this is so, why then the noise about the 12 German divisions in W.E.U. and N.A.T.O.?

Times, 8/6, 21 juillet 1955 [OED]

Le terme mentionné peut également correspondre à la reprise d'un terme précédemment employé :

(37) But why "kick sorter"? Because it sorts out electrical "kicks" or impulses according to their amplitude - more than 16,000 of them in each of 100 channels and at speeds up to 1,250 pulses per second.

Economist, 767/2 (Advt.), 7 septembre 1957 [OED]

(38) When I told a distinguished psychologist that I differed from his view of the universe, he answered, "Why universe? Why should it not be a multiverse?"

Chesterton, New Jerusalem viii, 1920, p. 163 [OED]

(39) "What are you going to film?" asked Jacquie.

"Well, it doesn't really matter, so long as it's innocuous," I said.

"Why innocuous?" asked Sophie.

"I don't want to take any risks [...]"

G. Durrell, A Zoo in my Luggage, 1960, p. 120-121

On notera que l'interrogation peut porter sur le choix du terme employé ou sur son énonciation, la distinction n'étant pas nécessairement pertinente (Why universe? peut s'interpréter comme "Why do you say universe?»). On retrouve cette ambiguïté (ou indétermination) quant à la véritable portée de la question dans d'autres types d'interrogatives en $w h y^{23}$. Certaines questions sont explicites à cet égard :

(40) Peter: I met her here, about a year ago. Edward: At one of Lavinia's amateur Thursdays? Peter: A Thursday. Why do you say amateur? Edward: Lavinia's attempts at starting a salon.

T.S. Eliot, Cocktail Party i. i, 1950, p. 37 [OED]

23. Dans l'exemple suivant, "Why? " équivaut à "Why do you ask if I'm serious?": Hugh eyed me for a moment. "Are you serious?" he inquired.

"Well, I was joking, but it would make a wonderful site for a zoo. Why?" I asked. (G. Durrell, A Zoo in my Luggage, 1960)

Comme le remarque G. Deléchelle (1989, p. 248) : «Il n’est [...] pas toujours facile avec les questions why? de distinguer le dit et le dire, le contenu énoncé et son énonciation. On sait qu'il n'y a pas beaucoup de différence entre demander à quelqu'un d'expliquer et lui demander de s'expliquer, entre questionner et mettre en question, entre demander des raisons et demander raison, entre cause et mise en cause.» 
(41) All France has learnt about the bands of "leather-jackets". Why are these young rowdies called "leather-jacket"? Certainly leather jackets seem to be the uniform for young American delinquents.

New Statesman, 180/1, 15 août 1959 [OED]

La forme interro-négative s'interprétera à nouveau comme une proposition concernant le choix d'un terme (au sens large), ce choix pourra éventuellement venir en opposition à un choix précédemment proposé :

(42) He said if a jig band could be a big success in Paris why not a fellow like you. New Yorker, 22/3, 7 octobre 1939 [OED]

(43) This was before the GAA (Gay Activists' Alliance - why not GAY? I suppose Gay American Youth would have been agist).

Maledicta iii, 1980, p. 249 [OED]

\subsection{Le cas de "Why should/would... ? "}

Les interrogatives en "Why should... ?» correspondent à un type intermédiaire entre les interrogatives du type: "Why + proposition finie?» et celles du type "Why + base verbale?". En effet, le marqueur -ed du modal should permet, comme la base verbale, de revenir au niveau de la mention de la notion complexe considérée (ou lexis). Le fait de mettre en suspens le repérage énonciatif du préconstruit ${ }^{24}$ permet au locuteur de mettre en évidence la distance qu'il établit entre lui et cette relation et d'exprimer ainsi son «rejet» du préconstruit. Mais l'emploi de should permet également de marquer de façon explicite ${ }^{25}$ l'existence de cette relation préconstruite (soit parce qu'elle renvoie à un événement avéré, soit parce qu'elle renvoie à une prise en charge subjective antérieure). Ainsi, tout en se repositionnant en un lieu (point de départ de la bifurcation) d'où le choix est encore possible (choix entre $\mathrm{p}$ et $\mathrm{p}$ ', ou entre I et E), la mémoire de l'existence ${ }^{26}$ du choix antérieur (préconstruit) est conservée.

Avec ce type d'interrogative, il existe une certaine variation possible dans le caractère polémique de l'énoncée ${ }^{27}$, mais comme pour "Why + base verbale?", la désactualisation du préconstruit entraîne facilement

24. Là encore, l'interrogative travaille sur un préconstruit (même si la préconstruction peut être fictive).

25. Contrairement à ce qui se passe avec la base verbale.

26. On perd cependant le type de positivité initiale de la relation. Celui-ci est récupérable par le contexte ou la situation et éventuellement par les propriétés primitives des notions lexicales en œuvre dans l'énoncé. Pour l'importance du contexte dans la reconstruction du préconstruit, je renvoie à mon article de 1997.

27. À ce sujet, je renvoie également à mon article (1997) qui développe ce point. 
vers une interprétation polémique ou rhétorique, confortant ainsi le décalage. Je rappelle ici brièvement les trois types de préconstruit auxquels peut renvoyer une interrogative en "Why should...?»:

- Le préconstruit (qui est toujours à reconstruire) possède une positivité de type Qnt (c'est-à-dire qu'il renvoie à un "état de fait» : état, propriété du sujet ou événement avérés) :

(44) "Some people think I'm a public menace. Actually, I'm a client, that is, if you're Cordelia Gray. Don't people ever tell you..."

Cordelia, unreasonably, was disappointed. Why should she have supposed that he was different from other male clients? She finished the sentence for him: "That it's an unsuitable job for a woman? They do, and it isn't."

P.D. James, The Skull Beneath the Skin, 1982, p. 8-9

(45) There might be something she herself could do to help. And even if not, it was impossible just to board the launch and set off as if the scene had never happened. She tried to silence the small resentful voice which protested that it couldn't have occurred at a more inconvenient time. Was she never to be allowed to get off the island? Why should she always have to be the one to act as universal social worker? But it was impossible to ignore such distress.

P.D. James, The Skull Beneath the Skin, 1982, p. 315

- Le préconstruit possède une positivité de type Qlt (c'est-à-dire qu'il value, d'une façon ou d'une autre, la validation de la relation) :

(46) [...] Then she said:

"Why don't you leave?"

She must have seen his incomprehension. She said again:

"Why don't you leave, leave this place, leave her?" Her voice, as always, was low but harsh, almost expressionless. He stared at her, wild-eyed under the dripping hair.

"Leave Clarissa! Why should I? What do you mean?"

P.D. James, The Skull Beneath the Skin, 1982, p. 85

- Le préconstruit peut également correspondre à un jugement épistémique (sur de l'avenir envisagé ou du passé à reconstituer) :

(47) "Unless Eliza Marley told him on the way here."

"Why should she tell Digby Seton? It's not a likely topic for conversation on the drive home. [...]"

P.D. James, Unnatural Causes, 1967, p. 77

(48) "Suppose she died. What would you do then?"

"Why should she die? She isn't sick, is she? She never said anything to me about being sick. What's wrong with her?"

P.D. James, Unnatural Causes, 1967, p. 88

Ce type de contexte hypothétique permet l'apparition d'interrogatives en "Why would... ?». La différence entre ces deux types d'interrogatives tient, on s'en sera douté, à la différence de modal. Le modal 
$\operatorname{shall}^{28}$ permet de localiser une occurrence situationnelle par rapport à un repère subjectif, d'où son utilisation privilégiée lorsque l'on veut maintenir la trace de l'existence d'un préconstruit et de son origine subjective. De son côté, will ${ }^{29}$ permet de marquer la conformité de l'occurrence situationnelle considérée au centre organisateur de la notion incarnée par la relation prédicative. Il suppose donc une adéquation entre la délimitation Qnt de l'occurrence et sa délimitation Qlt (ce qui n'est pas le cas avec shall).

Dans les interrogatives en "Why would... ? ", le marqueur -ed positionne le préconstruit sur un plan fictif (d'où l'affinité avec les contextes hypothétiques), will apporte l'idée d'adéquation entre la délimitation Qnt de l'occurrence (c'est-à-dire ici de l'occurrence situationnelle supposée) et sa délimitation Qlt, et l'interrogative en why marque la recherche de ce qui constitue cette délimitation Qlt. Selon la valeur contextuelle prise par will, la recherche sera orientée vers les motivations possibles du sujet (valeur "radicale» de volition) ou les raisons logiques, objectives (valeur "épistémique» de prédiction) qui permettraient de confirmer l'hypothèse en question.

Pour illustrer ces différentes possibilités et faire ressortir la différence entre "Why would... ? " et "Why should... ? ", on peut reprendre les exemples (47) et (48), en remplaçant should par would:

$\left(47^{\prime}\right)$ "Why would she tell Digby Seton?"

(48') "Why would she die?"

L'énoncé (47') équivaut à une recherche des motivations du sujet "she» : les raisons pour lesquelles elle aurait voulu en faire part à Digby. On voit ressortir ici la valeur de volition de will avec la présence à la fois de téléonomie et de valuation subjective. Il s'agit là de rechercher la délimitation Qlt qui serait en adéquation avec l'occurrence situationnelle de la relation (cette occurrence étant virtualisée, rendue fictive en tant que simple hypothèse). On voit bien ici la différence avec l'énoncé en should où la motivation du sujet (son agentivité/intentionnalité) n'intervient aucunement. En effet, en (47), si volition il y a, elle concerne la source subjective de l'hypothèse ${ }^{30}$ (et non le sujet de la relation prédicative), comme le montre la glose: "Pourquoi voulez-vous (qu' elle le lui ait dit) ? ", où "vouloir » n'a pas son sens à proprement parler volitif ${ }^{31}$,

28. Cf. Blanvillain 1998.

29. En ce qui concerne l'analyse du fonctionnement de will, je reprends ici celle développée par É. Gilbert (1998).

30. Qui n'est pas le locuteur, mais généralement son interlocuteur.

31. Sur les questions en français du type "pourquoi veux-tu que... ?», cf. Milner \& Milner 1975. 
mais marque toujours l'origine subjective de l'hypothèse. On pourrait également penser, pour ces interrogatives, aux tournures familières du type: "Pourquoi voulez-vous qu'elle aille le lui dire?» ou «Pourquoi irait-elle le lui dire?", où "aller" marque la distance que le sujet aurait (eu) à parcourir pour valider la relation et donc la distance qui le sépare de cette validation, marquant ainsi le caractère improbable de l'hypothèse aux yeux du locuteur.

Dans l'énoncé (48'), les propriétés primitives du verbe die écartent l'interprétation radicale du modal et nous orientent vers sa valeur de prédiction: quelle pourrait être la cause de son éventuel décès? On est à la recherche de ce qui peut justifier cette prédiction fictive sur l'avenir ${ }^{32}$.

Si l'on reprend maintenant le cas de la valuation positive, en effectuant la même manipulation :

(46') "Why would I leave Clarissa?"

la différence d'interprétation avec l'énoncé en should est très claire. En (46') le locuteur demande quelles seraient ses raisons, sa motivation personnelle pour vouloir quitter Clarissa (selon son interlocuteur). Avec should, ce qui est en cause ce sont les raisons de l'interlocuteur pour émettre une telle suggestion.

On voit donc que le fonctionnement est différent de celui des interrogatives en "Why should...?" ". Avec would, le marqueur -ed positionne la relation prédicative considérée sur un plan fictif, ce à quoi s'ajoute le fait que will suppose une adéquation entre les délimitations Qnt et Qlt de l'occurrence: les interrogatives en "Why would... ?" sont donc parti-

32. La prédiction ne porte pas nécessairement sur de l'à-venir :

"[...] What about the Major, Watts? He's a strange fellow."

"I've been thinking about that. It can't have been him, sir!"

"Really! Why not?"

"Why would he need to fire five times? He was an army pistol champion. He could have killed him with one shot." (Spoken English Corpus, University of Lancaster)

Il est intéressant de noter que bien que renvoyant à du passé, aucune marque d'antériorité n'est associée à would (mon corpus ne possède d'ailleurs aucun exemple de "would have v-en", bien que les anglophones acceptent de rajouter have-en sur des exemples de ce type). Ceci s'explique peut-être par le fait qu'avec "Why would... ?» le locuteur envisage la relation prédicative comme une simple hypothèse, sans insister (contrairement à "Why should... ?", que l'on trouve d'ailleurs facilement associé à un marqueur aspectuel) sur son caractère préconstruit.

33. Je remercie ici tous les anglophones qui ont accepté de répondre à mes tests concernant ces interrogatives en "Why should... ?" et "Why would... ?", et tout particulièrement ma collègue R. Dilys, dont les commentaires toujours circonstanciés ont beaucoup aidé ma réflexion. 
culièrement adaptées aux cas où le locuteur accepte de partir de l'hypothèse proposée par son interlocuteur (même s'il la rejette ensuite). Avec should, le marqueur -ed ne positionne pas la relation sur un plan fictif, il la décroche de la situation en la ramenant à une simple lexis ${ }^{34}$. D'autre part, shall ne suppose aucune adéquation entre les délimitations Qnt et Qlt de l'occurrence, d'où le fait que les interrogatives en "Why should... ?" soient plus facilement interprétées comme rhétoriques.

Le fonctionnement différent du marqueur -ed avec would et should explique également le fait que would puisse difficilement remplacer should quand le préconstruit renvoie à un "fait» (état, propriété, événement avérés) $)^{35}$.

Ce qui a été dit sur la différence de fonctionnement entre les interrogatives en "Why would... ?" et celles en "Why should...? " ne doit cependant pas laisser penser que seules ces dernières puissent être polémiques, comme le montrent bien les deux exemples suivants :

(49) "Did you wait for him?" "Wait, nothing!" said Cullam hotly. "Why would I?" R. Rendell, Best Man to Die xii, 1969, p. 117 [OED]

(50) 'They'll know I haven't got a licence. I'll tell them I can't drive. They can see I'm not strong enough to lift a big boy like that." Because Benet wasn't answering she began to drum with her heels. "Why would I take him? I don't like children. I didn't take him, I didn't and you can't make me say I did! How dare you say I took him!"

R. Rendell, The Tree of Hands, 1984, p. 106-107

34. Cette distinction peut être mise en parallèle avec celle établie, pour le français, par Culioli (1992, p. 19-21) entre si (qui marque une "assertion fictive») et que (qui marque une "désassertion").

35. Cela ressort clairement des tests mentionnés plus haut. Sans pouvoir développer longuement ce point, on pourra tout de même reprendre l'exemple (44): Why should she have supposed that he was different $[\ldots]$ ? Avec should, on comprend immédiatement qu'elle a effectivement supposé qu'il était différent ("She did. Why?»). Tandis que pour avoir would, il faut nécessairement un contexte hypothétique. Par exemple: elle n'a rien supposé de tel, et on s'interroge sur ce qui aurait pu la motiver pour le faire; ou encore: on ne sait pas si elle a ou non supposé quoi que ce soit, mais on en fait l'hypothèse et c'est alors l'interlocuteur qui s'interroge sur ce qu' auraient pu être ses motivations. Mais dans le contexte fourni par l'exemple (44) (qui ne permet pas une interprétation de type hypothétique), would est impossible. Et il en va de même pour tous les exemples de notre corpus, sauf un qu'il serait trop long d'analyser ici (et pour lequel je me contenterai de signaler la présence d'une hypothétique en if dans le contexte immédiat) :

Brick: I married you, Maggie. Why would I marry you, Maggie, if I was...? Margaret: Brick, don't brain me yet, let me finish! [...] (T. Williams, Cat on a Hot Tin Roof, 1957). 
En effet, on comprend aisément la possibilité d'une polémique lorsque le sujet de la relation est identifié à l'énonciateur : l'hypothèse confere à celui-ci une motivation dont lui seul peut rester juge.

Je conclurai sur les interrogatives argumentatives en résumant leur fonctionnement de la façon suivante: en interrogeant (de façon plus ou moins rhétorique) sur $\mathrm{p}$ (sur la raison de la validation, effective ou envisagée, de l'occurrence), on conclut en $\mathrm{E}$ (valuation négative de l'occurrence $)^{36}$. Dans ce cas, le but de l'interrogative est d'ouvrir la brèche fournie par le décalage instauré par why et de consacrer ainsi le hiatus entre les deux types de délimitations de l'occurrence.

\subsection{Why ? et what for?}

Je terminerai cette partie sur why interrogatif par quelques remarques comparatives concernant les interrogatives en "What for...? " " On pourra commencer par souligner la parenté de ces deux types d'interrogatives. Toutes deux peuvent questionner le «but» (pour why, cf. l'exemple (17), déjà mentionné). Les réponses aux interrogatives en "What for...?" suivantes montrent bien cette idée d'aboutissement notionnel :

(51) "What's this for?"

"For wearin'. Better than a bikini, isn't it?"

H. Kershaw, Elsie Tanner Fights back, p. 28 [Deléchelle 1989]

(52) "What do you want a guitar for? Are you going to play the International or something on it on board your ship?" Yvonne said. [...]

"To sing with. To give people the lie with maybe."

M. Lowry, Under the Volcano, 1947, p. 327

(53) And what for? so he could frame pictures of his grandkids!

H.V. Kershaw, Elsie Tanner Fights back, p. 73 [Deléchelle 1989]

36. Cf. Culioli 1990a, p. 121-122: "Dans l'interrogation rhétorique, on fait comme si on avait recours à autrui, mais la mélodie le montre clairement, on n'a pas demandé d'information. La stabilisation s'effectue par la sortie en E. On remarquera au passage que si l'on partait de E (n’est-il pas généreux ? ! - Bien sûr qu'il est généreux), l'interrogation rhétorique ferait aboutir en I ». Cette citation s'applique aux interrogatives fermées, mais on voit qu'on aboutit à une conclusion similaire pour les interrogatives ouvertes en why, si ce n'est que E correspond à la valuation négative de l'occurrence: l'interprétation rhétorique d'un énoncé du type Why did you do that? sera "You shouldn't have done that " (où "shouldn't" marque la valuation négative de la relation < you / do that $>$ ).

37. Je suis partie de l'étude de G. Deléchelle (1989, p. 232-245) à qui j'emprunte plusieurs exemples. 
Toutes deux peuvent interroger sur la «cause». Comme le montrent les exemples suivants, les interrogatives en «What for...? ? ne sont pas limitées à l'expression du «but ${ }^{38}$ :

(54) She came over and put her hand on my shoulder.

He beat me when you not here, I say. [...]

What he beat you for? she ast.

For being me and not you.

A. Walker, The Color Purple, 1983, p. 66

(55) $[\mathrm{He}]$ had been arrested the night before. What for? Importuning in a lavatory.

C. P. Snow, Corridors of Power, p. 285 [Deléchelle 1989]

Il faut toutefois remarquer que les contraintes sont sans doute plus fortes pour ces dernières, qui lorsqu'elles s'interprètent comme question causale semblent systématiquement porter sur un type particulier de verbes ${ }^{39}$, où l'idée d'origine notionnelle est "préformatée" (comme "beat» et "arrest» dans nos exemples). On pourra ajouter que les deux types d'interrogatives peuvent porter sur les motivations du sujet (on l'a déjà vu pour "Why...? ", et nous y reviendrons en ce qui concerne "What for...?"), et que toutes deux peuvent être polémiques. On le sait déjà pour "Why...? ", en voici seulement deux exemples illustratifs pour "What for...?" :

(56) What's that shrug for? What the hell are you shrugging like that for?

J. le Carré, Tinker, Tailor, Soldier, Spy, p. 160 [Deléchelle 1989]

(57) "Where's the other tractor?" they had demanded.

"Buried," said Big Joe.

"Buried!" cried the machine agent "What the hell for?"

"For eternity, brother," said Big Joe. "Unless you bring a shovel to dig her out."

R. Ross Annett, "Its' Gotta Rain Sometime", in More Stories from Western Canada, 1980, p. 87

Dans ce dernier exemple, Big Joe joue évidemment sur les valeurs de for, qui intervient ici dans l'expression de la durée et non du but ${ }^{40}$.

Si on peut à l'évidence rapprocher les interrogatives en "What for... ? " des interrogatives en "Why...? ", il faut également signaler ce qui les distingue. En effet, si avec "What for...? " l'idée de hiatus est

38. Dans ce cas, for ne représente plus «l'aboutissement notionnel du procès, mais bel et bien, en une sorte de renversement de la relation finale, son origine notionnelle " (cf. Gilbert 1999, p. 112, citation concernant la valeur «causale» de for).

39. Dont j'ai déjà parlé à propos de l'exemple (16), en référence à É. Gilbert, ibid.

40. Mais qui peut toujours se représenter métalinguistiquement comme l'expression d'un hiatus (par la construction d'un espace de validité sur la classe des instants), cf. l'article d'É. Gilbert dans ce recueil. 
permanente, du fait même de la présence du marqueur for, on pourra rappeler, comme on l'a vu au cours de cette étude sur le marqueur why, que ce dernier laisse ouverte la possibilité d'une adéquation entre délimitations quantitative et qualitative de l'occurrence, c'est-à-dire d'une stabilisation de son rapport Qnt/Qlt. L'étymologie de why me paraît d'ailleurs très éclairante à cet égard : l'OED indique en effet que why est l'instrumental (ou le datif) de what, gouverné par les prépositions to et for ou utilisé seul (le locatif étant where). Or, on sait que si to ${ }^{41}$ et for marquent tous deux une "visée", seul le premier est compatible avec l'idée d'objectif atteint et d'adéquation des délimitations Qnt et Qlt de l'occurrence.

Cette importance plus marquée du "décalage» avec les interrogatives en "What for... ? ", du fait de la permanence du "hiatus " exprimé par for (alors que why est étymologiquement lié aussi bien à to qu'à for), peut s'illustrer sur quelques exemples. Prenons :

(58) A thought flew across Gabriel's mind.

"Perhaps that was why you wanted to go to Galway with that Ivor girl?" he said coldly.

She looked at him and asked in surprise: "What for?"

Her eyes made Gabriel feel awkward. He shrugged his shoulders and said: "How should I know?"

$$
\text { J. Joyce, "The Dead " }
$$

Gabriel propose une identification (cf. "that was why...») de la motivation de son interlocutrice (sa raison de vouloir aller à Galway avec la fille Ivor), identification qui comblerait le hiatus et expliquerait l'occurrence, lui donnerait un sens, tandis que celle-ci recrée au contraire le hiatus avec "What for?", marquant ainsi sa surprise et la distance à laquelle elle se trouve d'une telle idée ("She [...] asked in surprise»).

L'exemple (59) peut sembler plus surprenant:

(59) "Sit down,"

"What for?"

"Because you're going to faint, that's what for."

H.V. Kershaw, Elsie Tanner Fights back, p. 128 [Deléchelle 1989]

41. Cf. Gilbert 1999, p. 104 : « to est potentiellement compatible avec l'idée d'un objectif atteint, et donc d'une adéquation entre délimitations quantitative et qualitative de la relation prédicative, c'est-à-dire avec une stabilisation du rapport Qnt/Qlt. For, au contraire, [...] maintient systématiquement un décalage entre les deux types de délimitation, interdisant par là même toute forme de stabilisation et maintenant du même coup la relation dans un statut de simple occurrence quantitative. Alors qu'avec to il peut y avoir à la fois occurrence situationnelle et notionnelle de la relation, avec for il ne peut y avoir qu'occurrence situationnelle. »

42. Exemple cité par E. Cottier à propos de "make» (pour Her eyes made Gabriel feel awkward). Cf. Cottier 1992, p. 106. 
En effet, la plupart du temps lorsqu'il y a identification d'une raison, c'est why qui est employé ("That's why...", cf. l'exemple (58) ainsi que les exemples (7) et (8) précédemment mentionnés). De plus, la présence de "because»" ${ }^{43}$, qui permet de combler le hiatus, pourrait également faire prévoir "that's why». Je pense que l'on peut fournir l'explication suivante à ce cas particulier: on est parti de l'interrogation "What for? " qui laissait clairement entendre que le locuteur ne voyait aucune raison de s'asseoir. Or son interlocuteur a une bonne raison de le lui demander (il a les moyens de combler le hiatus), il répond donc avec «because», mais reprend le "what for» employé précédemment par son interlocuteur pour répondre au plus près à cette question plutôt polémique.

On a vu que l'idée de hiatus était elle-même associée à l'idée de visée, d'objectif à atteindre et donc de téléonomie. Or, il semble que cet aspect soit particulièrement important dans le fonctionnement des interrogatives en "What for...?». Ce type d'interrogative est d'ailleurs le plus souvent associé à l'expression du "but», ainsi qu'à l'idée d' «intentionnalité». G. Deléchelle ${ }^{44}$ souligne qu'une "question en What for? est peu plausible avec un prédicat n'impliquant pas un certain degré de contrôle, d'intentionnalité » du fait que ce type de questions est particulièrement adapté «aux énoncés se rapportant aux motivations et à la finalité de l'action ". Il est intéressant à ce propos de s'arrêter sur l'association des interrogatives en "Why... ?» et "What for... ? » avec un verbe d'état.

Il semble en effet y avoir contradiction à interroger sur l'origine ou le but d'un état dont la caractéristique est de correspondre à un intervalle ouvert sans début ni fin. De plus, on a alors un fonctionnement compact du prédicat, or dans ce cas,

il semble que l'on soit d'emblée dans le qualitatif - d'emblée au centre, et ce sans que soit véritablement mise en œuvre la construction d'une

43. On pourrait sans doute poser l'hypothèse que "because» marque une adéquation entre les délimitations qualitative et quantitative de l'occurrence, à la différence de for qui, on l'a rappelé, marque un hiatus (un décrochage). On peut à l'appui de cette hypothèse concernant because rappeler les exemples du type des énoncés (23) et (24) où "because» constitue la totalité de la réponse à une question "Why... ?" et suffit à marquer la stabilisation du rapport Qnt/Qlt de l'occurrence. L'interrogative en why construit un décalage entre les délimitations qualitative et quantitative de l'occurrence en ce qu'elle questionne ce qui fonde qualitativement l'occurrence situationnelle considérée. La réponse en Because! fait disparaître le décalage en faisant savoir que l'occurrence est bien fondée qualitativement, même si ce qui la fonde n'est pas précisé. Autrement dit, because marque l'identification de l'occurrence situationnelle au centre organisateur de la notion incarnée par la relation prédicative, c'est-à-dire l'adéquation de ses délimitations Qnt et Qlt, ce qui fait de l'occurrence une occurrence notionnelle.

44. Cf. Deléchelle 1989, p. 237. 
occurrence. $[\ldots]$ avec de tels procès, on ne peut obtenir d'occurrence si ce n'est par le biais d'un support externe à savoir le sujet ${ }^{45}$.

Il semble donc là encore y avoir contradiction entre ce centrage qualitatif immédiat, mais sans découpe notionnelle possible et l'interrogation en «Why... ?» ou en "What for...? ? dont le but est précisément de structurer notionnellement l'occurrence. D'où le côté bizarre des énoncés du type:? Why are you ugly/blonde/so small? ou ?? What are you ugly/ blonde/so small for?, dont les prédicats (exprimant un état permanent ou une propriété intrinsèque ${ }^{46}$ ) ne supposent a priori ni cause, ni but et renvoient à un domaine pré-centré.

L'association de "Why...? ? ou de "What for...? ? avec un verbe d'état n'est pourtant pas impossible:

(60) "What does this theology do for you? After all, you spent three years on it. Teach you how to live? Answer some of the questions?"

"What questions?"

"The big questions. The ones there's no sense in asking. Why are we here? What happens when we die? Have we really free will? Does God exist?"

"No, it doesn't answer questions. It's like philosophy, it tells you what questions to ask."

P.D. James, A Certain Justice, 1997, p. 305

(61) What are we alive for? Why?

D. Lessing, Each, p. 136 [Deléchelle 1989]

Dans ce cas, la contradiction se résoud dans l'interprétation métaphysique $^{47}$ de la question qui renvoie alors à une cause "première», ou à un but "ultime" puisqu'elle équivaut à la recherche de ce qui fonde la "réalité", de ce qui la justifie et la structure.

Il est également possible qu'aucune réponse ne soit attendue (d'autant qu'aucune ne semble concevable - autre que métaphysique, comme on l'a déjà dit). Ce type de question rhétorique permet de mentionner un fait tout en en déplorant l'existence:

(62) Why should quiet ruminants like you and I have been born in such a ghastly age?

C.S. Lewis, Lettre du 22 mars 1966, p. 179 [OED]

45. Cf. de Vogüé 1987, p. 59.

46. Il est toujours possible de rétablir une cause externe ou un but, mais il semble que l'on passe alors à une propriété extrinsèque. Une question du type: Why are you so small (when your parents are so tall) ?envisage la propriété be small comme un héritage génétique externe, ce qui réintroduit la possibilité d'une cause. De même pour imaginer une question interrogeant sur le but, il faut que la caractéristique soit temporaire (décoloration: What are you blonde for?).

47. Ou naïve (question d'enfant qui suppose une cause ou un but à toute chose), les deux se rejoignant peut-être... 
Mais dès lors que l'état est temporaire ou la propriété extrinsèque au sujet, rien ne s'oppose plus (au contraire) à l'existence d'une cause ou d'une raison logique:

(63) "Then why is Tuppy short of cash? Didn't he inherit them?" "Not by a jugful."

P. Wodehouse, Much Obliged, Jeeves vi, 1971, p. 57 [OED]

(64) "What's a boffin?"

"The man from Farnborough. Everybody calls them boffins. Didn't you know?" "Why are they called that?"

"Because they behave like boffins, I suppose."

N. Shute, No Highway iii, 1948, p. 61 [OED]

Lorsque le prédicat correspond à une caractéristique du sujet (action répétée qui peut être considérée comme une propriété ou une attitude caractéristique du sujet), comme dans l'exemple suivant:

(65) Catherine Beddington turned to her and said: "[...] She'd have been fine with someone who was clever and could stand up to her."

Trudy said: "Preferably male. She didn't like women. And you are clever. You got a Two-One, didn't you? Why the hell do women always underrate themselves?"

P.D. James, A Certain Justice, 1997, p. 230

selon le degré d'agentivité que l'on accorde au sujet de ce type d'énoncés, on peut penser qu'il s'agit soit d'une question rhétorique ${ }^{48}$ (le locuteur se contentant de déplorer un état de fait), soit d'un reproche fait aux femmes (que l'on considère alors comme responsables de leur façon d'être). Et comme le montrent les exemples suivants, dès que l'état peut être clairement assimilé à une attitude (volontaire) ou qu'apparaît la moindre intentionnalité, l'interrogation peut se reporter sur les motivations du sujet (et correspondre à la mise en cause de la source - agentive, intentionnelle - de l'état en question) :

(66) Why have you got those roses in your hair? You look like the dog's dinner.

C. L. Anthony, Touch Wood ii, 1934, p. 66 [OED]

(67) No matter that Israel is the only country in the region that is a democracy [...] or that no principal action in Israel goes unscrutinized or uncriticized. Why then is the American press so quick to accept mistatements and unfounded claims put forth by the Arabs without so much as a demur?

B. Tepper, «LETTERS: Was the Press Taken In?», Time Magazine, 4 novembre 1996, p. 6

Si donc les interrogatives en "Why...? ? et en "What for...?" associées à des prédicats compacts sont toutes deux soumises à certaines

48. On est proche dans ce cas de l'exemple (45). 
contraintes d'interprétation et si la motivation subjective peut réapparâ̂tre très vite avec why, il semble bien que l'aspect «intentionnel » ne disparaisse jamais avec "What for... ? ». En effet, même avec l'énoncé (61), "l'interrogatif semble impliquer une volonté, un contrôle» ". J'ajouterai que cette intentionnalité peut renvoyer au sujet de l'énoncé ou à une instance extérieure, comme le montrent les exemples suivants :

(68) "What are you in this state for?": "Pourquoi est-ce que tu t'es [ou on t'a] mis dans cet état?"

[Deléchelle 1989]

(69) "What's the door locked for?"

"It just locked itself."

J. Updike, Run Rabbit Run, p. 10 [Deléchelle 1989]

Ces quelques constatations me semblent conforter le lien, précédemment mentionné, entre hiatus, visée, intentionnalité, motivations subjectives et questions en "What for... ?». Rappelons que l'intentionnalité et la motivation subjective ne sont pas absentes des interrogatives en why, loin de là, mais on peut cependant noter que ces notions n'y sont pas attachées de façon nécessaire.

G. Deléchelle a remarqué que "What for...?" n'était pas compatible avec la négation: * "What didn't you do it for? ", " What for not? " (tandis que "Why didn't you do it? " et "Why not?" sont possibles). L'explication que je propose à ce phénomène est encore liée à l'idée de hiatus que véhicule for. En effet, pour construire un hiatus, il faut deux points : la position correspondant à l'occurrence considérée (qui sert d'ancrage) et la position visée (s'il s'agit de but) ou la position de départ (s'il s'agit de cause). Or, la négation ne permet pas la construction d'un point d'ancrage ("You didn't do it» ne peut constituer un ancrage puisque la négation d'une positivité Qnt marque l'absence ou l'inexistence ${ }^{50}$ : «il n'y a pas de... » ou "p n'est pas le cas » $\left.{ }^{51}\right)$. L'absence du point d'ancrage empêche donc la construction du hiatus et par là même l'emploi de for (ce qui n'est pas le cas avec why, qui on l'a vu - s'il instaure également un décalage - n'est pas incompatible avec une adéquation des délimitations Qnt et Qlt de l'occurrence et ne renvoie donc pas systématiquement à l'existence d'un hiatus). Il en va de même pour les impossibilités suivantes: * "What that shrug for?" (mais "What's that shrug for?" est possible) ou * "What worry for?", dans la mesure où la mention

49. Cf. Deléchelle 1989, p. 237.

50. À moins que l'absence constitue elle-même une forme de positivité (quand elle s'interprète par exemple comme un refus), mais je n'ai pas trouvé d'exemple attesté pour illustrer ce point.

51. Cf. Franckel \& Lebaud 1990, p. 215. 
(base verbale ou syntagme hors relation prédicative) ne permet pas non plus de construire un point d'ancrage.

\section{Why interjectif-exclamatif}

Je me contenterai ici de donner rapidement les conclusions de mon analyse concernant ce type d'emploi de $w h y^{52}$. Why $!^{53}$ distingue deux zones qui sont en relation d'altérité, et marque le passage d'une zone à l'autre. Mais, là encore, why n'exprime pas un hiatus inhérent à l'occurrence, mais un décalage qui peut n'être que temporaire et disparầtre par la suite. Si l'on examine tous les types d'occurrences de why en contexte exclamatif, on s'aperçoit qu'il apparaît toujours en réaction à un déficit au niveau de la délimitation Qlt de l'occurrence (indétermination ou inadéquation). L'occurrence en question peut renvoyer à un élément textuel (ce que vient de dire l'interlocuteur, par exemple) ou situationnel (la situation observée ou un élément de cette situation). Cette occurrence (à laquelle « réagit " why) constitue la première «zone » délimitée. Le «déficit» au niveau de la délimitation Qlt de l'occurrence peut correspondre à de nombreux cas différents. Je ne prendrai ici que l'exemple classique où le déficit correspond à la surprise ${ }^{54}$ du locuteur (c'est-à-dire à l'inadéquation de l'occurrence par rapport à la situation ou à l'attente du locuteur). On peut illustrer une des multiples possibilités correspondant à ce cas par l'exemple suivant :

(70) (Mae enters bearing aloft the bow of a young lady's archery set)

Mae: Brick, is this thing yours?

Margaret: Why, Sister Woman - that's my Diana Trophy. Won it at the intercollegiate archery contest on the Ole Miss Campus.

T. Williams, Cat on a Hot Tin Roof, 1957, p. 29

Ce déficit entraîne un parcours (marqué par $w h-y$ ). Ce qui suit why correspondra à la deuxième zone délimitée et constituera une issue au parcours, selon les cas: entrée en I (par identification comme dans l'exemple précédent), centrage, ou refus d'entrer en I (comme dans l'exemple suivant):

(71) "Me take the harness off him!" my mother said, surprised. "Why, I wouldn't touch that mad thing with a forty-foot pole."

Coast to Coast, 1941, p. 167 [OED]

52. Cela pour maintenir cet article dans des limites de taille raisonnables. Mais je me propose de présenter cette analyse dans le détail dans un prochain article sur quelques tournures exclamatives en anglais contemporain.

53. Ce fonctionnement de why exclamatif est à mettre en parallèle avec la forme schématique proposée pour mais, en français, par Culioli (cf. Culioli 1992, p. 10).

54. Qui peut être agréable ou non. 
Le décalage entre les deux zones délimitées correspond donc à une différence (différenciation/non-identification) de positionnement par rapport au domaine notionnel associé à l'occurrence considérée.

Why marque encore une fois un décalage et l'issue au parcours qu'il introduit peut consacrer ce décalage (refus d'entrer en I, c'est-à-dire refus de fonder qualitativement l'occurrence ou de reconnaître son bienfondé) ou au contraire le réduire (ou tendre à le réduire) : entrée en I (ou mouvement vers I), voire centrage.

\section{Conclusion}

Nous avons vu, à travers l'étude des différents emplois de why, que ce dernier, en tant que représentant du "fondement qualitatif " de l'occurrence, introduit automatiquement un décalage entre la délimitation quantitative de l'occurrence et sa délimitation qualitative. Cependant, why ne pose pas ce décalage comme inhérent à l'occurrence, et n'est pas incompatible avec l'idée d'adéquation des délimitations Qnt et Qlt de l'occurrence considérée. En effet, l'instanciation de la variable wh-permettra éventuellement de faire disparaître le décalage en levant l'indétermination qualitative de l'occurrence, lui (re)donnant ainsi un statut d'occurrence notionnelle (la "cause " attestera du bien-fondé de l'occurrence). Il n'en reste pas moins que why, en marquant l'indétermination qualitative de l'occurrence considérée, déstabilise le rapport Qnt/ Qlt de cette occurrence, ouvrant ainsi la brèche à toutes les remises en cause. Le décalage qu'il exprime ne se limite pas toujours à la distance qui sépare l'ignorance de la connaissance : il peut également correspondre au hiatus existant entre l'attente du locuteur et la situation, exprimant ainsi l'inadéquation de l'occurrence ou sa valuation subjective négative.

En tant qu'il exprime une indétermination qualitative, why marque donc un décalage qui peut être réduit une fois l'indétermination levée, mais ouvre également la voie à l'irréductibilité entre « désir» et « réalité».

\section{Références bibliographiques}

Adamczewski H. \& Delmas C. (1982, $4^{\mathrm{e}}$ éd. 1993), Grammaire linguistique de l'anglais, Paris, Armand Colin.

55. On a vu qu'il pouvait s'agir aussi bien de la "cause» (ou origine notionnelle) que du «but» (ou aboutissement notionnel). 
Blanvillain O. (1997), "Pourquoi voulez-vous que "Why should...?" soit rhétorique? Opérations et indices contextuels ", in Co-texte et Calcul du sens (Actes de la table ronde tenue à Caen les 2 et 3 février 1996), C. Guimier (éd.), Caen, Presses universitaires de Caen, p. 181-195.

Blanvillain O. (1998), "Shall: visée et prise en charge énonciative», communication au colloque international du Cercle belge de linguistique Les verbes modaux dans les langues germaniques et romanes (les 10-11-12 décembre 1998 à Anvers).

COTTIER E. (1992), «L'opérateur causatif make et ses traductions françaises : dissymétrie avec le français faire», Linguistique contrastive et traduction, 1, p. 81-125.

Culioli A. (1982), «Rôle des représentations métalinguistiques en syntaxe ", communication présentée à la session plénière du XIII ${ }^{\circ}$ Congrès International des Linguistes, Tokyo.

Culioli A. (1990a), "Formes schématiques et domaine", Pour une linguistique de l'énonciation. Opérations et représentations, Gap, Ophrys, "L'homme dans la langue», t. 1, p. 115-126.

Culioli A. (1990b), "Autres commentaires sur bien», Pour une linguistique de l'énonciation. Opérations et représentations, t. 1, p. 157-168.

Culioli A. (1992), «De la complexité en linguistique», Le Gré des Langues, 3, p. 8-22.

DELÉCHELLE G. (1989), L'Expression de la cause en anglais contemporain, Thèse de Doctorat d'État, Université de la Sorbonne Nouvelle, Paris III, (dactyl.).

Franckel J.-J. \& Lebaud D. (1990), Les Figures du sujet. À propos des verbes de perception, sentiment, connaissance, Gap, Ophrys, "L'homme dans la langue».

GILBERT É. (1998), "À propos de will», communication au colloque international du Cercle belge de linguistique Les verbes modaux dans les langues germaniques et romanes.

GILBERT É. (1999), "De quelques emplois de for», in Les Opérations de détermination. Quantification/Qualification, A. Deschamps \& J. Guillemin-Flescher (éds), Gap, Ophrys, "L'homme dans la langue», p. 101-107.

GRESSET S. (1984), "Which/that, marqueurs de relatives", Cahiers de recherche en grammaire anglaise, t. II, p. 201-268. 
MiLNER J. et MiLnER J.-C. (1975), «Interrogations, reprises, dialogue», in Langue, discours, société - Pour Émile Benveniste, J. Kristeva, J.-C. Milner \& N. Ruwet (éds), Paris, Seuil, p. 122-148.

MiLNER J. (1983), Fragments linguistiques sur l'interrogation, Thèse de Doctorat d'État, Université Denis Diderot Paris VII (dactyl.).

Oxford English Dictionary (2 ${ }^{\mathrm{e}}$ éd. 1992), Oxford University Press (CD-ROM).

Quirk R., Greenbaum S., Leech G. N. \& Svartvik J. (1985), A Comprehensive Grammar of the English Language, Longman.

VoGÜÉ S. DE (1987), «Aspect: construction d'occurrences », T. A. informations, 1, p. 47-61. 